\title{
Predicting operating room case load: An aid to resource allocation
}

\author{
Elizabeth M. Geary' ${ }^{1}$, Martin Goldberg ${ }^{2}$, A. G. Greenburg ${ }^{3}$, Thomas E. Johnson, J r. ${ }^{4}$ \\ 1. Smithfield, RI, USA. 2. Madison, AL, USA. 3. Department of Surgery, Brown University, Providence, RI, USA. \\ 4. Department of Information Systems and Decision Sciences, College of Business, University of South Florida, Tampa, \\ FL, USA.
}

Correspondence: Martin Goldberg. Address: Madison, AL, USA. E-mail: mgol.al@outlook.com

Received: July 4, 2013

Dol : $10.5430 /$ jha.v2n4p151

\begin{abstract}
Hospital patient bed utilization can reach $100 \%$ with an impact on elective surgery schedules. Analysis of the demand for beds created by elective surgical operations is desirable to manage overall resources under these conditions. For planning and allocating operating rooms, staff, beds and equipment on any given day, hospital administrators would benefit from an accurate prediction of the number of surgical cases that will be completed. Current scheduling techniques do not predict, for a given day in the future, the number of cases that will actually be performed.

A study was performed at a 247 bed hospital with 10 operating rooms. The operating rooms were available for reservation more than two weeks in advance. Both block scheduling and open time were available. Using reservation data with a simple Black Box model allows the prediction of the total number of cases to be performed up to two weeks in advance with $90 \%$ accuracy. The resultant predictive demand should allow for better resource planning for the Operating Suite as well as required post-op hospital patient beds.
\end{abstract}

\section{Key words}

Operating room, Scheduling, Forecasting, Predicting, Case load, Resource allocation

\section{Overview}

Full hospital occupancy causes disruptions in surgery schedules by forcing cancellations of elective procedures due to last-minute lack of beds. When this happens, patient and physician satisfaction, as well as markers and measures of quality assessment, all suffer. Staffing of people and equipment for this dynamically changing environment is very challenging. The hospital in this study, like many others, runs at, or near, full capacity most of the time. The need thus arises for more efficient handling of the projected demand for operations and operating suite room resources to allow for better allocation of people and equipment. The more accurate the case number prediction, the better the ability to schedule people and equipment in advance and provide more accurate input to the process that maximizes bed occupancy on a day-to-day basis. A tactical model was developed to meet this need. Using reservations made in the Operating Suite scheduling system, this model can predict, up to two weeks in advance, the expected number of cases that will be completed on any given day. 


\section{Operating room scheduling}

Creating a schedule for Operating Rooms to run efficiently and smoothly within the available scheduled hours is a very complex task. Having the correct equipment and instruments at hand, and to sustain efficiency, maintain budget and maximize revenues without under utilizing or over utilizing the Operating Rooms is crucial. It is important to have available staff: Nurse Anesthetist and/or Anesthesiologist for anesthesia plus Circulating, Scrub, Surgical Technician, Pre-Operative and Post Anesthesia Care (recovery room) nurses. Equally important is having enough in-house beds available for the surgical patients being admitted on the day of Surgery.

At this site, Operating Room scheduling for elective surgery is a balancing act for resources which can start as far out as six months and continue up to three days before surgery. The Operating Room scheduling system consists of the following types of time: individual surgeon block time, surgical group block time, service block time or open time for the initial scheduling of elective cases. Two weeks forward is the cut-off date for block booking by surgeons and groups. If they fail to schedule in their block, the time is considered "open," becoming available to any staff surgeon. The majority of cases are scheduled within two weeks of the actual surgical date, in originally scheduled open time or previous blocked time that has been released.

The scheduling system will calculate average case times for every planned surgical procedure. It will give a conflict check warning if there is not enough time or if specific equipment is not available for the planned Procedure. Some operating rooms may be restricted to certain procedures i.e. Cystoscopy, Open Heart or an urgent/emergency room. Urgent cases are scheduled within three days and emergencies within 24 hours of the procedure time.

The hospital faces challenges beyond the Surgical Services department. Paramount is making sure there are enough hospital beds available to prevent cancellation and future rescheduling of patient operations. Equally important is not having patients recovering in the Post Anesthesia Care Unit (a department not routinely open in off hours except for the recovery of emergency cases) and not paying overtime for staff. It is also essential to be prepared with enough staff for in house patient care needs without the cost of unexpected overtime.

\section{The predictive model}

One of the very basic tools in the analyst's toolbox is the "Black Box model" ${ }^{[1,2]}$. This model provides the means to analyze a system without actually knowing how it works. A Black Box model is a mathematical formula defined as Output = $\mathrm{f}$ (Input), where " $\mathrm{f}$ " is the function that describes the Black Box. In our case the Black Box is the complex function of the operating suite interacting with the entire hospital. The known input is the number of reservations made in advance and the known output is the number of cases actually performed. The scheduling system, the operating suites and their interaction are within the Black Box. How they actually interact is left without the need for formal analysis. The aim of the Black Box analysis is to develop a function that represents how the box transforms inputs into outputs. In this case, the input (Daily Scheduled Data), was combined with the output (Actual Cases Completed), and the data was mathematically analyzed to determine the functional relationship. This function, representing the effort of the Operating Suite, would then map values of Scheduled data into predicted Cases Completed.

The initial advanced prediction period for the model was chosen to be two weeks, the same length of time used by the operating room scheduling system when all open time slots are available to everyone. To cover this period of prediction, two weeks in advance, 14 functions were created mapping reservations to completed cases for each of the 14 days in advance that reservation data was available. A PC program was created to collect these data points (Number of Scheduled Cases and Actual Cases Performed) for each of the 14 day advanced periods. Over 30 data points for each of the 14 functions was derived by extracting the Scheduled and Actual data points for a fixed advanced period, and a PC curve fitting program was used to identify the function that was the best possible match. The curve fitting program used was 
Curve Expert ${ }^{[3]}$. It will fit over 30 functions to the data points presented and statistically rank the ability of the functions to match the collection of data points allowing for easy selection of the "best" fitting formula. Another PC program was created that, depending on the number of days in advance a prediction was requested, selected the proper function and used the formula for the function's expected value to predict the Cases Completed.

Based upon all data points initially collected, a typical curve fit is shown in Figure 1.

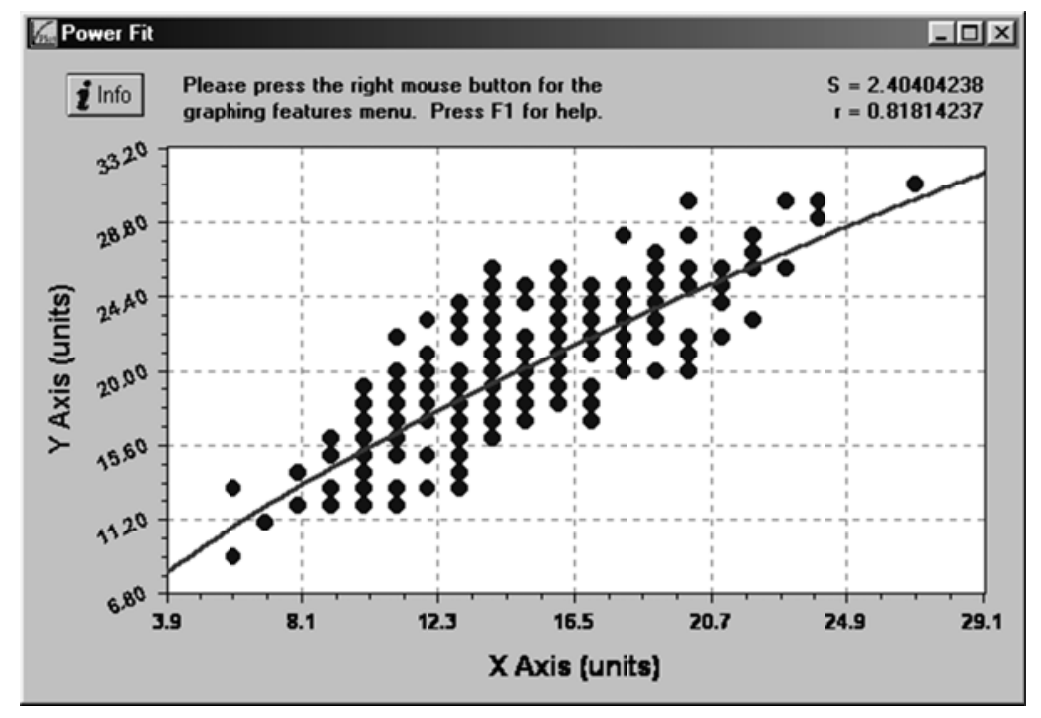

Figure 1. Typical curve fit

This model produced an accuracy of less than $65 \%$. This is about the same accuracy one would get using the scheduled numbers as expected number of cases completed. If used, it would provide case load numbers that would lead to understaffing of Operating Room personnel and underestimating the number of beds needed after all operations for the day are completed. To improve the accuracy of the model, a review of the data collected suggested modification of the functional mappings for the Black Box model, so additional data collection and model modification were initiated. In a separate analysis, undertaken to understand some of the variance noted, the difference in completed cases varied with the day of the week. Monday and Tuesday were the busiest. Wednesday seemed to be the slack day of the week. Thursday and Friday appeared moderate compared to Monday and Tuesday. These distributions are shown in Figure 2.

\subsection{The second model}

With one major exception in how the data was structured, the second model is very similar to the first. In the first model the collected data was aggregated; in the second model data for each day of the week was treated independently.

As before, the period for prediction was chosen to be two weeks. To cover this period, two weeks in advance for each weekday, 98 independent functions were created to allow the mapping of reservations to completed cases. Each function was derived from over 45 data points from about one year of data and the PC curve fitting program was used to get the best possible function that matched the data. The data produced 12 unique mathematical functions (see Table 1), each with variations described by the function's mathematical parameters. The PC prediction program was modified to select the proper function, obtain its defining parameters and use its expected value to predict the Cases Completed. The function selected by the PC program is the function derived from the day of the week for the desired estimate of cases completed and the number of days in advance the prediction is requested. 


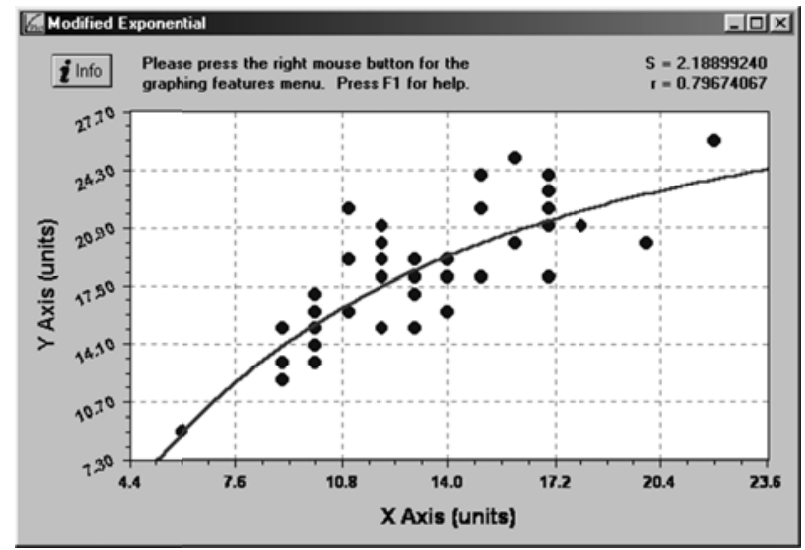

(a) Typical Monday data

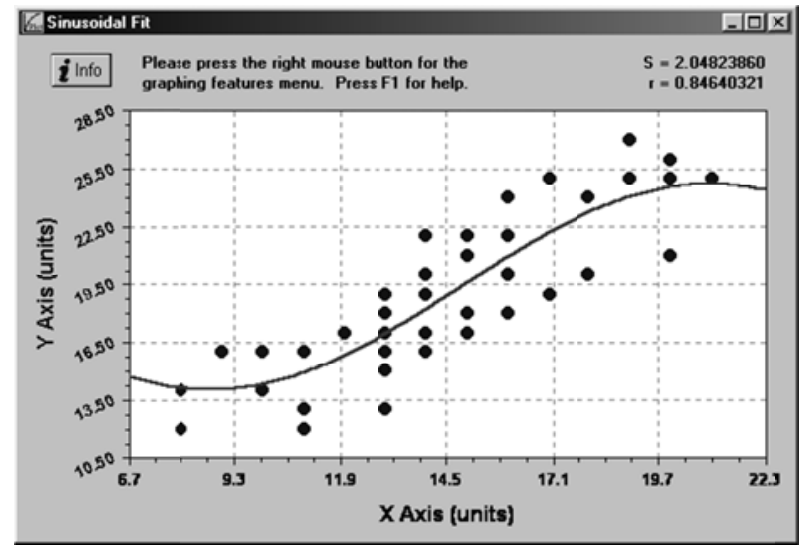

(c) Typical Wednesday data

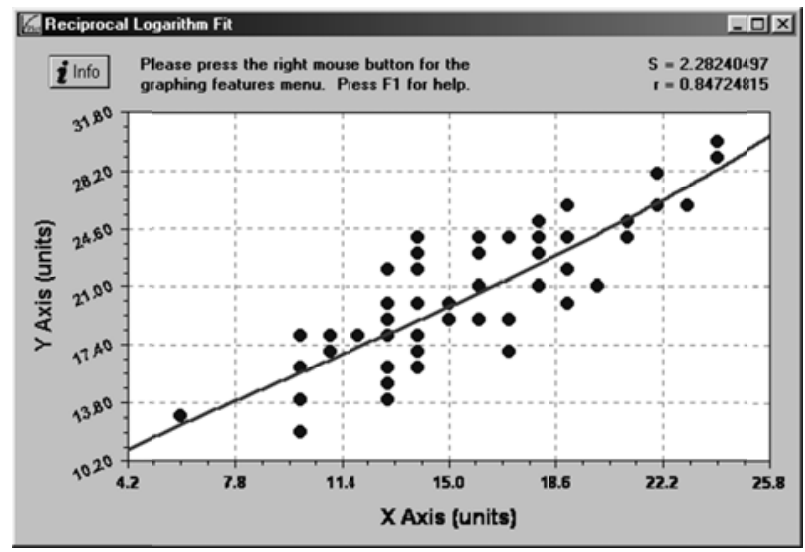

(b) Typical Tuesday data

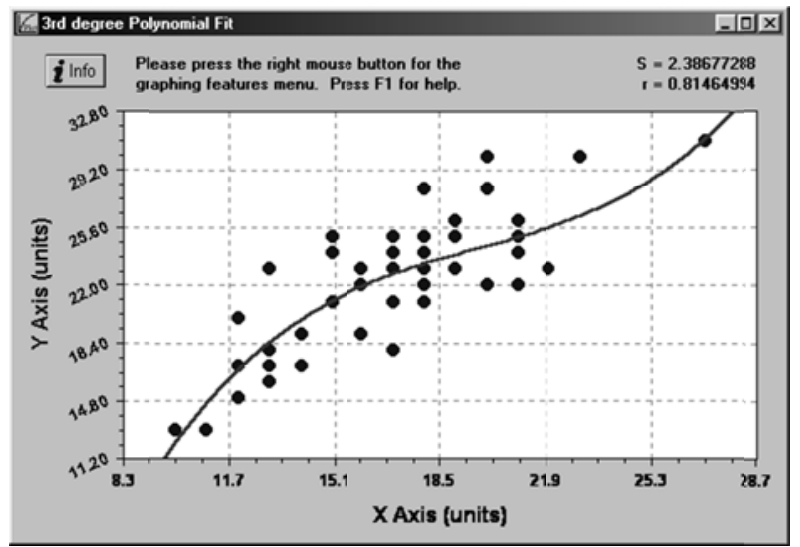

(d) Typical Thursday data

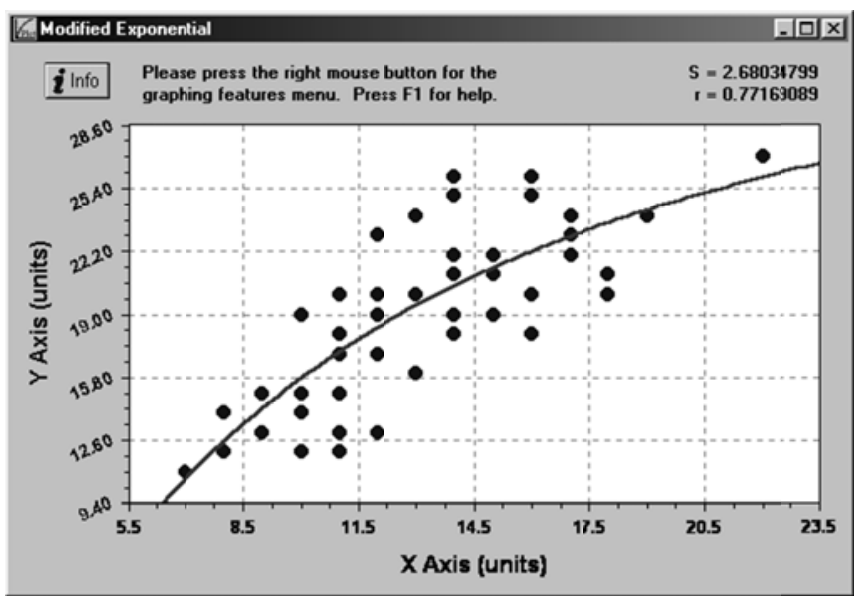

(e) Typical Friday data

Figure 2. Typical data of the week

To better understand the use of the model, consider the following sample operation of the prediction program to obtain an estimate for Friday based upon Tuesday's reservations, nine days in advance. First one enters the desired date for the estimate: Thursday 5/28. Then the day of the reservation count is entered: Tuesday 5/19. Next the current number of reservations for Thursday 5/28 known on 5/19 is entered, in this example " 17 ”. The Estimator then calculates an expected value of "23" total cases for Thursday 5/28. Had the number of reservations been seven, perhaps caused by a drop in demand, then the estimator would return " 18 ”. 
Table 1. Equations used by the model

\begin{tabular}{ll}
\hline Fit & Equation \\
\hline Linear & $\mathrm{y}=\mathrm{a}+\mathrm{bx}$ \\
Nth order Polynomial & $\mathrm{y}=\mathrm{a}+\mathrm{bx}+\mathrm{cx} \wedge 2+\ldots$ \\
Modified Exponential & $\mathrm{y}=\mathrm{a} * \exp (\mathrm{b} / \mathrm{x})$ \\
Logarithm & $\mathrm{y}=\mathrm{a}+\mathrm{b}^{*} \ln (\mathrm{x})$ \\
Reciprocal Logarithm & $\mathrm{y}=1 /\left(\mathrm{a}+\mathrm{b}^{*} \ln (\mathrm{x})\right)$ \\
Power & $\mathrm{y}=\mathrm{a}^{*} \mathrm{x}^{\wedge} \mathrm{b}$ \\
Geometric & $\mathrm{y}=\mathrm{ax} \wedge(\mathrm{bx})$ \\
Modified Geometric & $\mathrm{y}=\mathrm{ax} \wedge(\mathrm{b} / \mathrm{x})$ \\
Exponential Association & $\mathrm{y}=\mathrm{a}^{*}(1-\exp (-\mathrm{bx}))$ \\
Saturation-Growth Rate Model & $\mathrm{y}=\mathrm{a} \mathrm{a}^{*} \mathrm{x} /(\mathrm{b}+\mathrm{x})$ \\
Gaussian Model & $\mathrm{y}=\mathrm{a} * \exp \left(\left(-(\mathrm{b}-\mathrm{x})^{\wedge} 2\right)\left(2 * \mathrm{c}^{\wedge} 2\right)\right)$ \\
Hyperbolic & $\mathrm{y}=\mathrm{a}+\mathrm{b} / \mathrm{x}$ \\
\hline
\end{tabular}

Note: Where $\mathrm{y}$ is the estimated number of cases that will be completed, $\mathrm{x}$ is the number of reservations booked in advance and $\mathrm{a}$, $\mathrm{b}$ and $\mathrm{c}$ are constants for the regression programs produced by the Curve Fitting Program.

This example demonstrates the difference of about 30 percent between the estimate of cases to be completed from the scheduling system and from the Operating Suite Case Load Estimator. The scheduling system will, on average, provide an estimate that is within 30 percent and always below what actually happens. The Operating Suite Case Load Estimator will, on average, provide an estimate that is within 10 percent of actual and a slight over estimate. To validate the accuracy of the model, it was tested on both historical and contemporary, day-to-day numbers. Short weeks, those with Holidays, or school "breaks" were less accurately predicted. Additional work and analysis is required in this area of the model to improve its operation under these conditions.

\subsection{Validation of the model}

To validate the model it was exercised two ways. First it was used for a single test, in real time, to estimate completed case load three, four and five days in advance resulting in 100\% accuracy. This was done when the Operating Suite manager was to be elsewhere for a few days. The model was also statistically tested on a dataset consisting of 70 data points where the actual number of cases were recorded and contrasted to the models predictions. Because the data is non-parametric, the predicted distribution was compared to the actual distribution producing a Chi-Square of 1.00, resulting in a probability of 0.3173 with a resulting significance well below $0.05 \%$. This confirms that the two distributions are the same. In addition, a simple analysis of the differences ${ }^{[1]}$ showed an average difference of 2 , which is less than $10 \%$ inaccuracy. A similar analysis of the relationship between actual cases and reservations showed no relationship at all.

\section{Use of the literature}

To place this effort into a workable context, a literature search was undertaken. First, the current state of operating room models was checked and second, the state of Operating Room Load predictive models was determined.

Initially the search term "Operating room scheduling” was used. This search, and additional searches using refined terminology failed to find any articles specifically relating Scheduled Operations in the Operating Room to the Case Load outcome. Operating room planning and scheduling: A literature review ${ }^{[4]}$ is an excellent review of over 100 scheduling articles. However, it does not address the issue of predicting case load outcome from scheduling. The review has several good studies available that address scheduling but do not predict outcome or address the potential uses for this data. Other papers in the review address the application of operations research and pure probabilistic models to support the scheduling 
aspects of operating room interests. Finally, there were some discussions of data complications that had been overcome in these studies. Unfortunately none of these fit the particular question raised in this work.

Since the goal of this study was to develop, for hospital administrators, an efficient methodology to predict Operating Suite case load in the future, two weeks hence from any given day, the search moved from the more frequent applications into the relatively unknown predictive methods. To refine the search, the term "prediction" was added to the search keywords resulting in even fewer appropriate hits. Incorporating ideas involving the proper implementation and use of disaggregated data with reference to scheduling and outcome were not any more yielding.

\section{I mplications for the hospital}

The hospital can benefit from an accurate, advanced estimate of cases completed with a small increase in cost to collect, analyze and utilize Operating Room Schedule data. The increase in costs includes: daily data collection of cases completed, monthly data reduction and analysis, monthly updates to the estimating computer program and daily use of a hospital file server to store the program and data base. The cost of Information Technology staff must also be addressed. Tuning of the model requires the continuous re-calculation of the model functions to match changes in the resources within the complex operation of the Operating suite. As medical staff, techniques and technology change, the process of the Operating suite will also change. These changes must be factored into the model using newly collected data points and Black Box functions.

The increase in benefits includes: improved patient and physician satisfaction from fewer last minute cancellations, fewer crisis management events to locate beds in an over utilized hospital, better staff scheduling with less last minute shift extensions into overtime, and better utilization of the Operating Room suite. Combining these factors will provide lower cost from better staff scheduling and increased revenue from fewer case cancellations.

\section{Conclusion}

A study was performed at a 247 bed hospital with 10 operating rooms to determine whether the case load could be predicted two weeks in advance. Both block scheduling and open time were available. Using the reservation request data with a simple Black Box model allows for the prediction of the total number of cases to be performed two weeks in advance with 90\% accuracy. The resultant predictive demand for hospital patient beds provides data that could be useful in improving operating room staffing and hospital bed resource utilization.

\section{References}

[1] James T., McClave, P. G., Benson, Terry Sincich. Statistics for business and economics 10e. 10th ed. Upper Saddle River, N.J. Perrson Prentice Hall. 2008; 904.

[2] Giuseppe M. F. Di Roccaferrera. Operations Research Models for Business and Industry. 1964; Cincinnati: South-Western No. 966.

[3] Daniel G. Hyams. Curve Expert. 2005; Retrieved September 3, 2012, from http://www.curveexpert.net/.

[4] Brecht Cardoen., Erik Demeulemeester., Jeroen BeliÄen. Operating room planning and scheduling: A literature review, Department of Decision Sciences and Information Management, Katholieke Universiteit Leuven. Retrieved September 3, 2012, from https://lirias.kuleuven.be/bitstream/123456789/165923/1/KBI_0807.pdf. 